\title{
A New Simple Route to ZnS Quantized Particles with Tunable Size and Shape, and Size/Shape-Dependent Optical Properties
}

\author{
Pengfei $\mathrm{Hu}^{1 *}$, Yali $\mathrm{Cao}^{2}$, Yanyan $\mathrm{Lou}^{1}$, Bo Lu${ }^{1}$, Min Shao ${ }^{1}$, Jiansen $\mathrm{Ni}^{1}$, Meng $\mathrm{Cao}^{3}$ \\ ${ }^{1}$ Laboratory for Microstructures, Shanghai University, Shanghai, China \\ ${ }^{2}$ Institute of Applied Chemistry, Xinjiang University, Urumqi, China \\ ${ }^{3}$ School of Materials Science and Engineering, Shanghai University, Shanghai, China \\ Email: *hpf-hqx@shu.edu.cn
}

Received December 13, 2012; revised January 20, 2013; accepted January 27, 2013

\begin{abstract}
With the features of convenience and eco-friendly, the low-temperature solid-state reaction synthesis was successfully developed as a new approach to prepare quantum-sized ZnS nanocrystals. One major achievement is that the size and shape of ZnS nanocrystals can be tuned by adjusting the surfactant and its feed. The UV-Vis absorption spectra of quasi-spherical and one-dimensional quantum-sized $\mathrm{ZnS}$ nanocrystals all showed a blue-shift from the bulk counterpart, indicating large quantum confinement effects of $\mathrm{ZnS}$ nanocrystals. These $\mathrm{ZnS}$ nanocrystals all showed well-defined excitonic emission features. Contrastive studies on photoluminescence performances indicated that the band-edge emission experienced only the size-dependent quantum confinement effect, while the trap-state emission experienced the size- and shape-dependences. So we can design a purposeful synthesis route to $\mathrm{ZnS}$ nanocrystals with target luminescence emission performances.
\end{abstract}

Keywords: Solid-State Nanosynthesis; Tunable; Size- and Shape-Dependence; Optical Properties

\section{Introduction}

Owing to fascinating size-, and shape-dependent electronic and optical properties, quantized semiconductor structures have attracted increasing attention [1-3]. It is well known, for instance, that the band gap of semiconductor nanostructure increases as the particle size decreases when the dimension of nanocrystals approach to exciton Bohr radius, due to quantum-size confinement effect [4]. As one member of the important II-VI group semiconductors, zinc sulfide (ZnS, direct band gap of 3.7 $\mathrm{eV})$ motivated worldwide enthusiasms of scientists. Quantized ZnS (Q-ZnS) particles and Q-ZnS-based heterostructures have become essential light-harvesting and light-emitting components in modern technology such as imaging, diagnostics, biosensing, and display technologies [5-7]. Up to now, some methodologies for synthesizing Q-ZnS have been demonstrated [8-10]. Among most nanosynthsis techniques, surface-passivated technology in solution-process received relatively more interest $[3,4,8-11]$. It used surface-bonding ligands to repress the agglomeration of primary colloidal nanocrystals and obtain free-standing nanoparticles. However, this

"Corresponding author. methodology generally requires complicated manipulations including delicate control of solution composition, reaction atmosphere, $\mathrm{pH}$ value, reaction temperature and time, even of temperature ramp. Therefore, it is necessary to explore simple and convenient strategies to produce quantized structures with tunable size/shape and performance.

The low-temperature solid-state reaction synthetic approach provided a relatively simple and powerful method for controlling the size and shape of nanoparticles [12, 13]. In view of the advantages of low cost, convenience, and lack of pollution, we introduced it to synthesize the Q-ZnS firstly. We fulfilled the size-, and shape-control of Q-ZnS through surfactant-assistanted technique, and the tunable optical behavior.

\section{Experimental}

The manipulation for the synthesis of Q-ZnS through one-step solid-state reaction at room temperature was recorded and illustrated in Figure 1. The surfactants sodium dodecyl benzene sulfonate (SDBS) and cetyltrimethyl ammonium bromide (CTAB) were introduced to assist the synthesis, respectively. Zinc acetate dihydrate $\left(\mathrm{Zn}(\mathrm{OAc})_{2} \cdot 2 \mathrm{H}_{2} \mathrm{O}\right)$ powders were firstly blended 


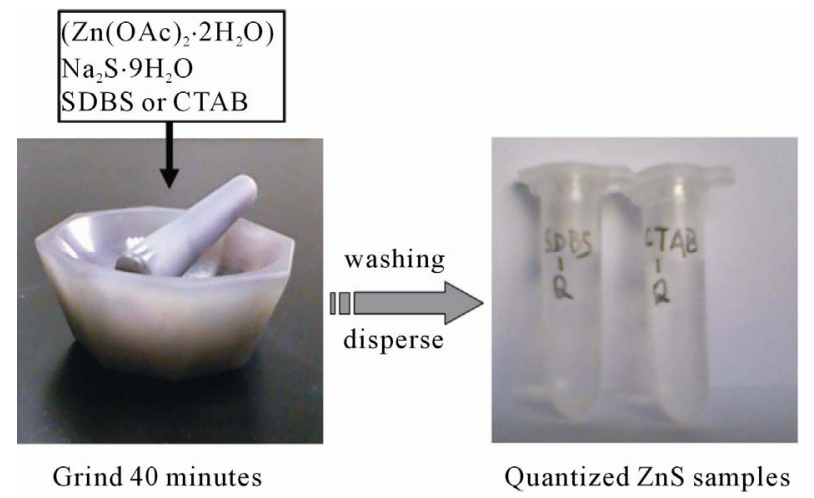

Figure 1. A convenient and effective recipe for quantized structures.

with an appreciable proportion of SDBS (Q-ZnS-i) or CTAB (Q-ZnS-ii) $\left(\mathrm{MR}_{\mathrm{SDBS}}\right.$ or $\mathrm{MR}_{\mathrm{CTAB}}=1: 5$, here, the molar ratio of additive $/ \mathrm{Zn}(\mathrm{OAc})_{2} \cdot 2 \mathrm{H}_{2} \mathrm{O}$ was designated as MR) and ground together for $10 \mathrm{~min}$ at room temperature, then sodium sulfide hydrate $\left(\mathrm{Na}_{2} \mathrm{~S} \cdot 9 \mathrm{H}_{2} \mathrm{O}\right)$ was added with 1:1 molar ratio between $\mathrm{Zn}(\mathrm{OAc})_{2} \cdot 2 \mathrm{H}_{2} \mathrm{O}$ and $\mathrm{Na}_{2} \mathrm{~S} \cdot 9 \mathrm{H}_{2} \mathrm{O}$. The mixtures were ground for about $30 \mathrm{~min}$, and centrifugation washed, respectively. Finally, the Q$\mathrm{ZnS}$ samples were dispersed in deionized water, respectively, and clear solutions were obtained for following characterization.

\section{Results and Discussion}

\subsection{Crystal Structures of $\mathrm{ZnS}$ Nanocrystals}

The X-ray diffraction (XRD) patterns for as-prepared Q-ZnS-i and -ii clearly indicates that they have cubic phase with lattice constant as cross-referenced to JCPDS 65 - 9585 card (Figure 2(a)). Three characteristic diffraction peaks at $2 \theta$ values of $28.7 / 28.9,47.4 / 47.4$, and
56.5/56.6 to Q-ZnS-i and -ii can be indexed to the crystal planes of (lll 111$),\left(\begin{array}{lll}2 & 2 & 0\end{array}\right)$ and ( $\left.\begin{array}{lll}3 & 1 & 1\end{array}\right)$. The cell constants were calculated to be $\mathrm{a}=\mathrm{b}=\mathrm{c}=0.5382 / 0.5375 \mathrm{~nm}$ from (111) peaks of Q-ZnS-i and -ii after refinement. They revealed that the lattice contractions of $\Delta \mathrm{a}=0.50 \%$ and $0.63 \%$ occurred against to the reported data $(\mathrm{a}=\mathrm{b}=\mathrm{c}=$ $0.5409 \mathrm{~nm}$ ), which implied the smaller sizes of ZnS particles and the high surface to volume ratio of nanocrystals. X-ray energy-dispersive spectroscopy (EDS) shown that the atom ratios of $\mathrm{Zn}$ to $\mathrm{S}$ are about 1:1.03 to Q-ZnS-i and 1:1.05 to Q-ZnS-ii, respectively (Figure 2(b)).

Additionally, the behaviors of SDBS and CTAB capping on the $\mathrm{ZnS}$ nanoparticles were identified by FT-IR spectra. The FTIR spectra of Q-ZnS-i and SDBS are compared in Figure 3(a). It is obvious that the Q-ZnS-i hold the characteristic peaks of sulfonate around 1210 $1025 \mathrm{~cm}^{-1}$ (denoted by the olive dotted ellipse in Figure 3(a)). In the FT-IR comparison of CTAB and Q-ZnS-ii, the transmission bands at 2920 and $2850 \mathrm{~cm}^{-1}$ in both are similar and respectively assigned to the asymmetric and symmetric stretching vibrations of methylene $\left(\mathrm{CH}_{2}\right)$ in the long alkyl chain, as well as that the transmission bands of Q-ZnS-ii in the ranges of $965-720 \mathrm{~cm}^{-1}$ and $1390-1490 \mathrm{~cm}^{-1}$ are close to that of CTAB (the olive dotted ellipses in Figure 3(b)).

As shown in Figure 4A and insets a-c, the Q-ZnS-i nanocrystals are the quasi-spherical nanoparticles. The average size of these nanoparticles is $2.5-3.5 \mathrm{~nm}$. The inset $\mathrm{c}$ of Figure 4A shows clear twin and stacking fault (SF) structures in a big nanoparticle. When CTAB was used as additive, the $\mathrm{ZnS}$ nanocrystals put up asymmetrical growth. As shown in Figures 4B, insets, and S1, near one-dimensional nanocrystals were recognized clearly, such as acicula-shaped nanocrystal (inset b in

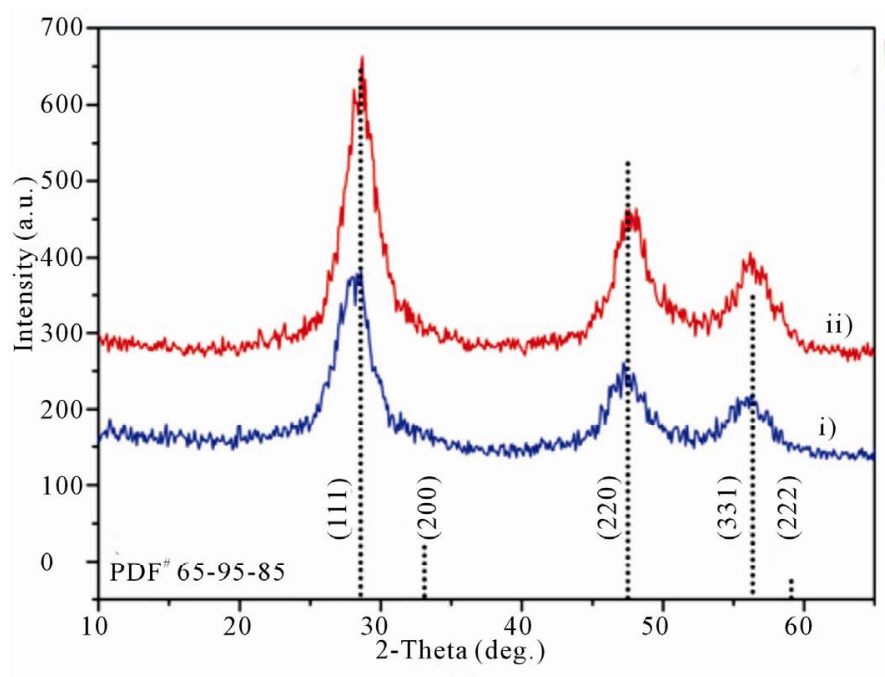

(a)

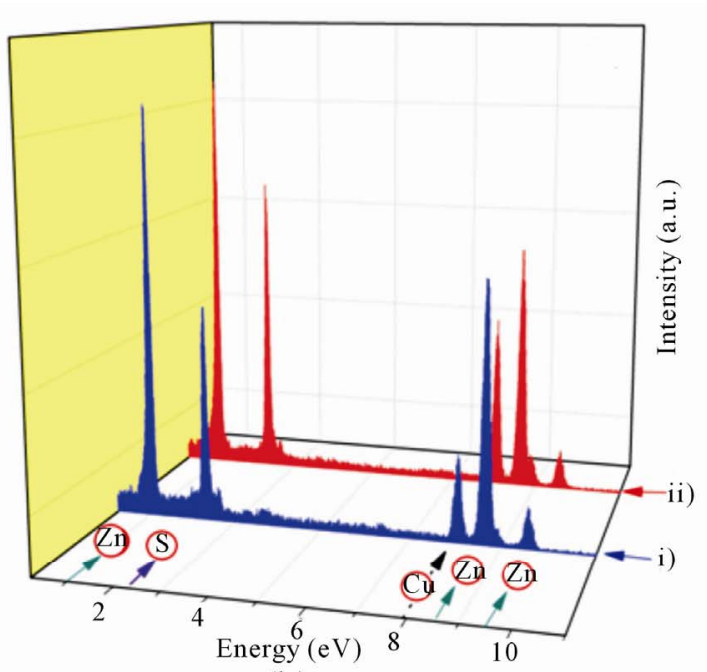

(b)

Figure 2. XRD patterns (a), and EDS spectra (b) of ZnS nanocrystals: Q-ZnS-i (blue curve-i), and Q-ZnS-ii (red curve-ii). 


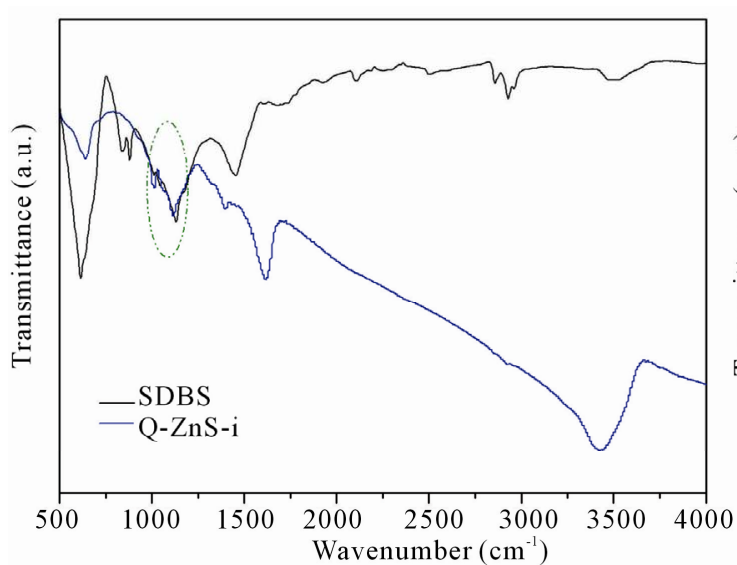

(a)

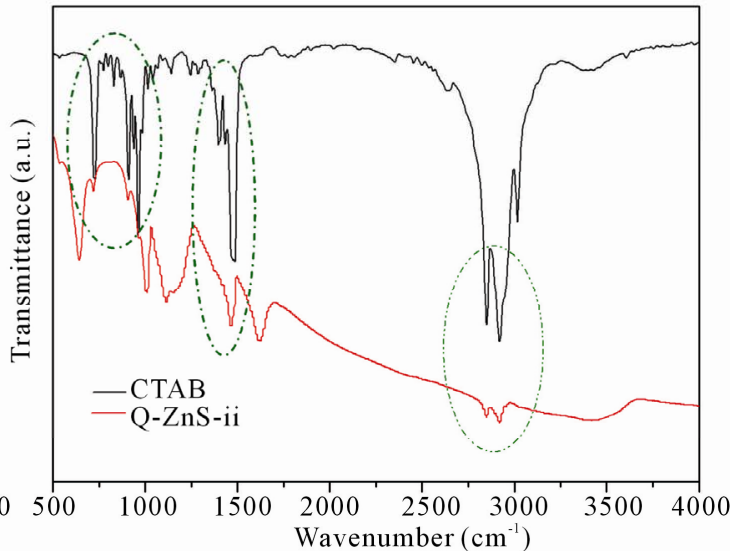

(b)

Figure 3. FT-IR spectra of SDBS and Q-ZnS-i (a), and CTAB and Q-ZnS-ii (b).
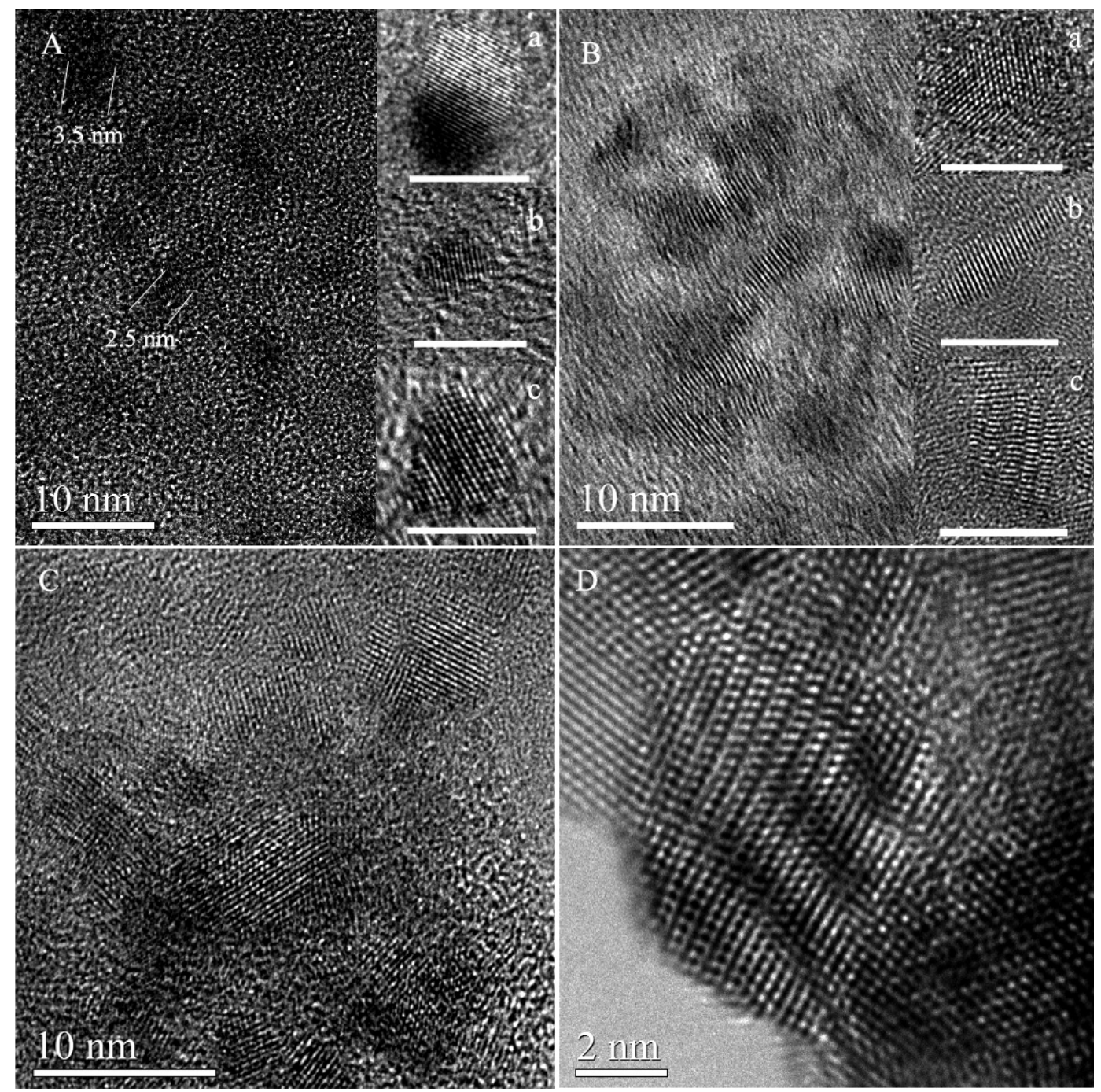

Figure 4. (A) TEM images of $3 \mathrm{~nm}$ quasi-spherical Q-ZnS-i nanocrystals synthesized with MRSDBS = 1:5; (B) TEM images of $3 \mathrm{~nm}$ near one-dimensional Q-ZnS-ii nanocrystals obtained with MRCTAB = 1:5; The insets a-c in panels $A$ and $B$ are HRTEM images of representative nanoparticles of Q-ZnS-i and -ii respectively (bar scale: $5 \mathrm{~nm}$ ); (C) TEM images of $7 \mathrm{~nm}$ quasi-spherical Q-ZnS-iii nanocrystals synthesized with MRSDBS = 1:10; (D) Higher resolution details of the Q-ZnS nanoparticles with wave-like structure. 
Figure 4B). It is worthwhile to note that some nanocrystals show the wave-like structure containing a large number of SF and dislocation at troughs (inset $\mathrm{c}$ in Figures $4 B, D$ and S1). We shall see in a forthcoming section that these novel structures can affect the photoluminescence properties of nanocrystals. In addition, to investigate the size control of particles in the solid-state reaction synthesis, $\mathrm{MR}_{\mathrm{SDBS}}$ was change to $1: 10$ to synthesize the Q-ZnS-iii. The Q-ZnS-iii nanocrystals are dominant with about $7 \mathrm{~nm}$-sized quasi-spherical nanoparticles (Figure 4C).

\subsection{Optical Properties of $\mathrm{ZnS}$ Nanocrystals}

\subsubsection{UV-Vis Spectral Characteristics}

Figure 5 shows the UV-Vis absorption spectera of the Q-ZnS-i and -ii. They almost remain the same shape which ends near $350 \mathrm{~nm}$ for about $3 \mathrm{~nm}$-sized nanocrystals. Each of spectra is the superimposition of a relatively well-defined band with peaks around 264 (Q-ZnS-i) or $259 \mathrm{~nm}$ (Q-ZnS-ii), an ill-defined broad absorption between 340 and $268 \mathrm{~nm}$ approximately, and a high energy intense absorption below $250 \mathrm{~nm}$ only displaying a tail of absorption pattern. These resolved superimpositions of experimentally obtained absorption curves imply the relatively wide size distribution of products. The band gap values were obtained from the optical absorption spectra by plotting $(\alpha h v)^{2}$ versus $(h v)$, and then extrapolating the linear region of $(\alpha h v)^{2}$ versus $(h v)$ on the en- ergy axis, as shown in inset of Figure 5. The band gap values were estimated to be $4.73 \mathrm{eV}$ for Q-ZnS-i, whereas it is $4.78 \mathrm{eV}$ for Q-ZnS-ii. The blue shift of the absorption band compared to bulk counterpart causes the band gap widening which indicates that the as-prepared Q$\mathrm{ZnS}-\mathrm{i}$ and -ii offer strong quantum confinement effect.

\subsubsection{Photoluminescence Studies}

Photoluminescence is a luminescence phenomena that occurs in luminescent materials which can be excited to a higher energy state termed "excited state" after absorbing light at a specific wavelength, and then re-emit light with a lower energy through recombination of electron and hole $[14,15]$. Due to high sensitivity and non-destructive character, the photoluminescence (PL) technique has been widely used to investigate electronic structure of the surface of semiconductor nanoparticles. Many reports illustrated the luminescence mechanism of $\mathrm{ZnS}$ nanoparticles using the well-known energy-level diagram (Figure S2). The valence band is considered as made up of hybridized s-p orbitals from sulfur and zinc atoms, while the conduction band is mainly consisted of the s-p antibond states of $\mathrm{ZnS}$. Theoretically, luminescence emission includes band-edge emission and trap-state emission which is divided into shallow-trap and deep-trap emission according to the difference of electron levels [16]. Trap-stated PL signals mainly originate in defects containing vacancies and interstitials of semiconductors. The defects can be categorized either shallow or deep level

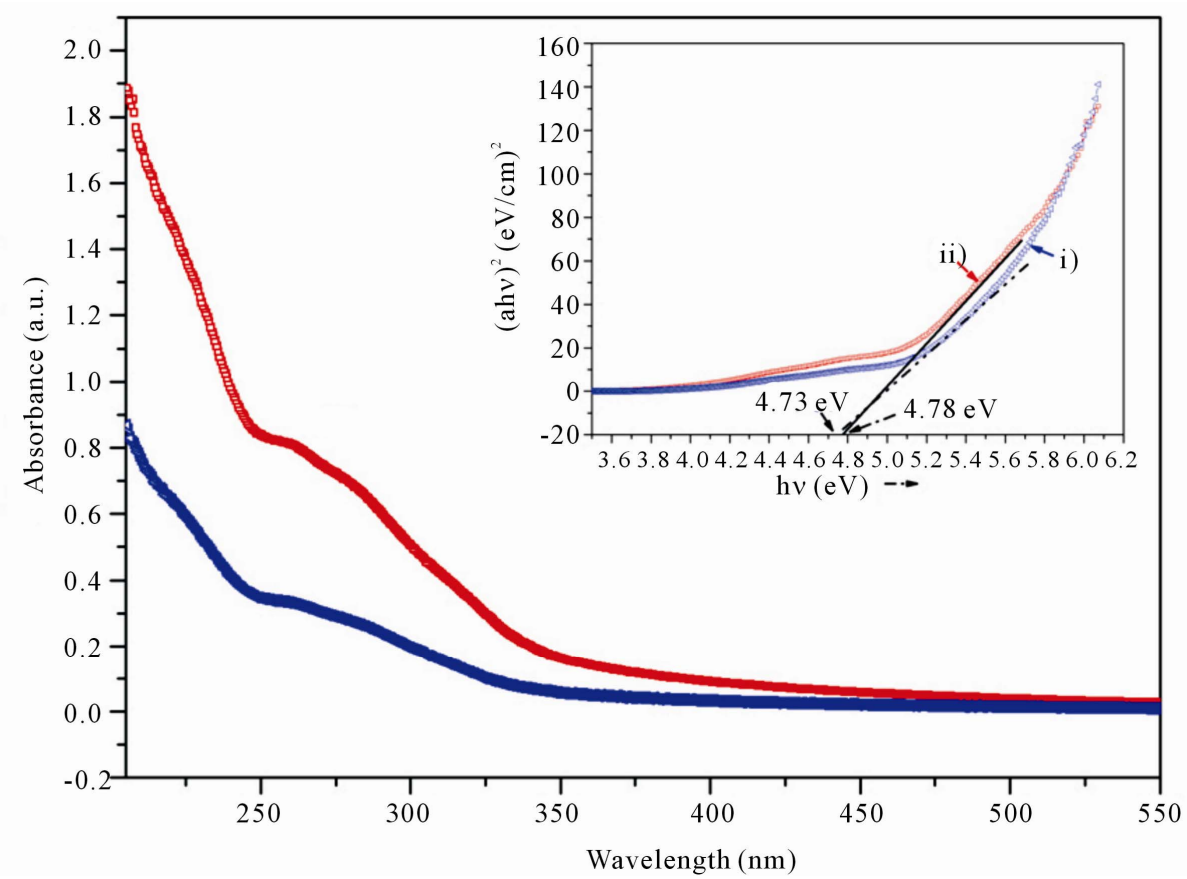

Figure 5. Comparison of UV-Vis spectra of $3 \mathrm{~nm}$ quasi-spherical Q-ZnS-i (blue symbol scatter-i) with one-dimensional Q-ZnS-ii (red symbol scatter-ii). The inset is the plots of $(\alpha \mathrm{hv})^{2}$ vs. (hv) for Q-ZnS-i (blue symbol scatter-i) and Q-ZnS-ii (red symbol scatter-ii). 
defect states. Shallow level traps, which are more spatially delocalized and lie near the conduction band or valance band edge, are more likely to participate in the radiative recombination. The emission occurs when a trapped electron recombines with a hole in the valence band or in some acceptor level such as zinc vacancies [17]. On the other hand, deep level traps tend to have a tendency to undergo non-radiative recombination by emitting phonons [16].

As far as we known, although many groups have put large efforts in studying the optical properties of $\mathrm{ZnS}$ nanocrystals, there have been few reports on the excitonic emission feature of $\mathrm{ZnS}$ nanocrystals, as well as the size- and shape-dependent emission characteristics. In most cases, only trap-state emission at around $450 \mathrm{~nm}$ was observed and discussed which is termed "tunneling luminescence" and controlled by defect and impurity, the size- and shape-dependent quantum confinement effects were neglected. Figure 6 shows the emission luminescence spectra of quasi-spherical (Q-ZnS-i) and one-dimensional (Q-ZnS-ii) $\mathrm{ZnS}$ nanocrystals, respectively, they were measured at $250 \mathrm{~nm}$ of excitation wavelength. The spectra all reveal the well-defined excitonic and trap-stated emission features. Many shallow trapped emissions indicated that the present nanocrystals occupied a great deal of effects.

Photoluminescence spectra of the quasi-spherical nanocrystals (Q-ZnS-i) showed broad violet emission peak centered at $342 \mathrm{~nm}$ accompanied by the broad asymmetry shoulder at $378 \mathrm{~nm}$, and smaller hump-like blue emission peaks at $422 \mathrm{~nm}$ and $439 \mathrm{~nm}$, and two weak shoulders at 456 and $500 \mathrm{~nm}$, respectively. We attribute the $342 \mathrm{~nm}$ peak to a band-edge emission. The shoulder centered at $378 \mathrm{~nm}$ is assigned to the overlap-ping peaks involving electronic transitions from conduction band to

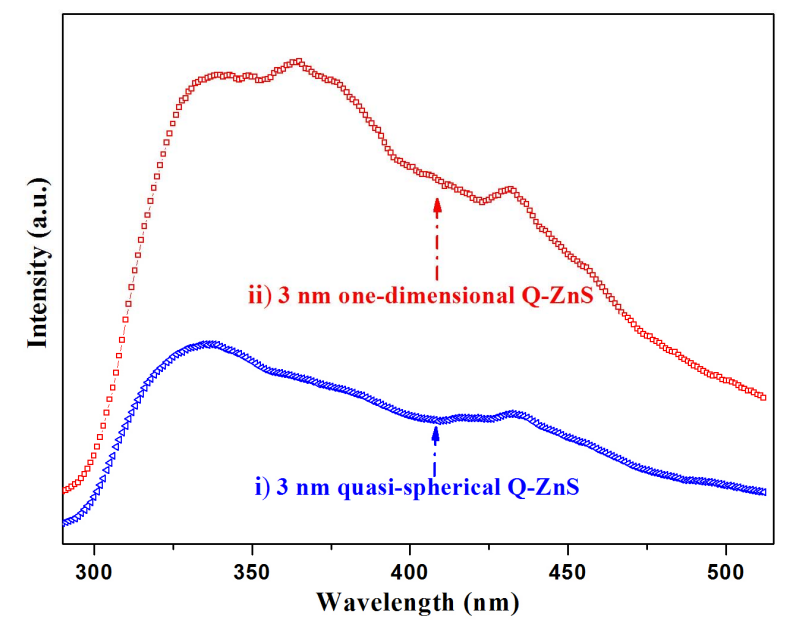

Figure 6. Comparison of photoluminescence spectra of the 3 nm quasi-spherical Q-ZnS-i (blue symbol scatter-i) with near one-dimensional Q-ZnS-ii (red symbol scatter-ii). interstitial sulfur and interstitial zinc to valance band. In addition, blue emission peaks at $422 \mathrm{~nm}$ and $439 \mathrm{~nm}$ are assigned to the energy level of sulfur vacancies with the holes from the valance band, and interstitial zinc from interstitial sulfur, respectively, while the last peak at 500 $\mathrm{nm}$ which can be attributed to recombination of electrons at sulfur vacancies to the holes at zinc vacancies. It is particularly worth noting that the emission band at 456 $\mathrm{nm}$ was attributed to dangling sulfur bonds at the interface of $\mathrm{ZnS}$ in this paper. Although the deep-trap emission at around $450 \mathrm{~nm}$ is ascribed to surface sulfur vacant sites in some literatures [18], we can exclude the peak at $456 \mathrm{~nm}$ from the trap-state emission because there is excess sulfur in this report as demonstrated by the EDS data.

The emission spectra of near one-dimensional $\mathrm{ZnS}$ (Q-ZnS-ii) showed a band-edge emission feature at 340 $\mathrm{nm}$, and high-intensity trap-state emissions about in range of $345-475 \mathrm{~nm}$ with well-defined peaks at 349 , 365 , and $432 \mathrm{~nm}$, and broad shoulder at $455 \mathrm{~nm}$. Compared to PL emission of quasi-spherical nanocrystals, the increase of intensity and shift of shallow-trapped emission implied that the near one-dimensional $\mathrm{ZnS}$ possess more defects, and the trap-state PL experimenced shapedependent quantum confinements. And the intensity of emission band at $455 \mathrm{~nm}$, which is ascribed to arise from the dangling sulfur bonds at the interface of $\mathrm{ZnS}$, slightly increased. On the other hand, the band-edge emission of $341 \mathrm{~nm}$ showed almost the same wavelength as that of quasi-spherical nanocrystals (342 nm). It is concluded in this work that the band-edge emission may be mostly dependent on size rather than shape. To further demonstrate the size-dependence of quantum confinement effect to band-edge and trap-state emission, PL spectrum of $7 \mathrm{~nm}$-sized quasi-spherical nanocrystals were got (Figure 7). The PL spectrum showed band-edge emission at $349 \mathrm{~nm}$, which was red-shifted $7 \mathrm{~nm}$ as that of $3 \mathrm{~nm}$ sized quasi-spherical nanocrystals. And the trap-state emissions all show varying degrees of red-shifts.

To estimate the applicability of approach adopted in this work, we introduce it to synthesize the CdS nanocrystals with SDBS. The $3 \mathrm{~nm}$-sized quasi-spherical CdS nanocrystals were successfully obtained (Figure S3).

\section{Conclusion}

In summary, quantum-sized $\mathrm{ZnS}$ nanocrystals with quasispherical and near one-dimensional shapes were synthesized through room-temperature solid-state reaction methodology. The synthetic procedure has the advantages of convenient operation, and low cost, and lack of pollution, and for mass-production and good applicability. The size- and shape-controls can be achieved through using different additives and adjusting the MR. The quan tum- 


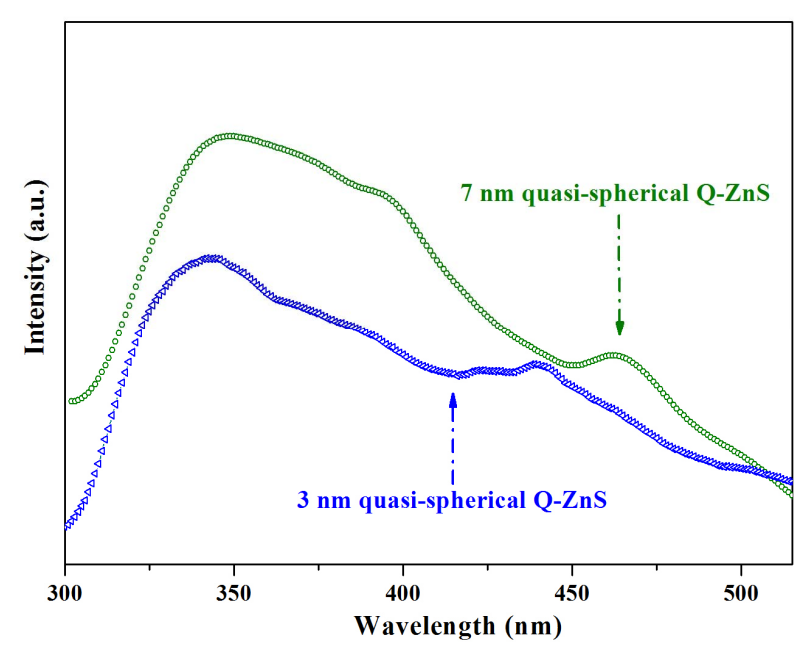

Figure 7. Comparison of photoluminescence spectra of the 3 nm quasi-spherical Q-ZnS-i (blue symbol scatter-i), and 7 nm quasi-spherical Q-ZnS-iii nanocrystals (olive symbol scatter-ii).

sized $\mathrm{ZnS}$ nanocrystals exhibited the quantum confinement effect and unique well-defined excitonic emission features. The band-edge PL emission showed the mostly size-dependent quantum confinement effect, but the trapstate PL experienced the size- and shape-dependences.

\section{Acknowledgements}

This work was funded by Shanghai University Innovation Foundation, National Nature Science Foundation of China (Grant No. 21101132), and National Nature Science Foundation of China (Grant No. 61006089).

\section{Supporting Information Available}

The characterization, the images of Q-ZnS-ii nanoparticles with one-dimensional and wave-like structures and CdS QDs, and Schematic energy level diagram.

\section{REFERENCES}

[1] L. E. Brus, "A Simple Model for the Ionization Potential, Electron Affinity, and Aqueous Redox Potentials of Small Semiconductor Crystallites," Journal of Chemical Physics, Vol. 79, No. 11, 1983, pp. 5566-5571. doi: $10.1063 / 1.445676$

[2] M. Nirmal and L. E. Brus, "Luminescence Photophysics in Semiconductor Nanocrystals," Accounts of Chemical Research, Vol. 32, No. 5, 1999, pp. 407-414. doi:10.1021/ar9700320

[3] X. G. Peng, L. Manna, W. D. Yang, J. Wickham, E. Scher, A. Kadavanich and A. P. Alivisatos, "Shape Control of CdSe Nanocrystals," Nature, Vol. 404, No. 6773, 2000, pp. 59-61. doi: $10.1038 / 35003535$

[4] V. I. Klimov, A. A. Mikhailovsky, S. Xu, A. Malko, J. A. Hollingsworth, C. A. Leatherdale, H.-J. Eisler and M. G.
Bawendi, "Optical Gain and Stimulated Emission in Nanocrystal Quantum Dots," Science, Vol. 290, No. 5490, 2000, pp. 314-317. doi:10.1126/science.290.5490.314

[5] X. Michalet, F. F. Pinaud, L. A. Bentolila, J. M. Tsay, S. Doose, J. J. Li, G. Sundaresan, A. M. Wu, S. S. Gambhir and S. Weiss, "Quantum Dots for Live Cells, in Vivo Imaging, and Diagnostics," Science, Vol. 307, No. 5709, 2005, pp. 538-544. doi:10.1126/science. 1104274

[6] T. H. Kim, K. S. Cho, E. K. Lee, S. J. Lee, J. Chae, J. W. Kim, D. H. Kim, J. Y. Kwon, G. Amaratunga, S. Y. Lee, B. L. Choi, Y. Kuk, J. M. Kim and K. Kim, "Full-Colour Quantum Dot Displays Fabricated by Transfer Printing," Nature Photonics, Vol. 5, No. 3, 2011, pp. 176-182. doi:10.1038/nphoton.2011.12

[7] C. L. Cowles and X. S. Zhu, "Sensitive Detection of Cardiac Biomarker Using ZnS Nanoparticles as Novel Signal Transducers," Biosensors and Bioelectronics, Vol. 30, No. 1, 2011, pp. 342-346. doi:10.1016/j.bios.2011.09.034

[8] K. E. Sapsford, I. L. Medintz, J. P. Golden, J. R. Deschamps, H. T. Uyeda and H. Mattoussi, "Surface-Immobilized Self-Assembled Protein-Based Quantum Dot Nanoassemblies," Langmuir, Vol. 20, No. 18, 2004, pp. 7720 7728. doi:10.1021/la049263n

[9] Y. W. Zhao, Y. Zhang, H. Zhu, G. C. Hadjipanayis and J. Q. Xiao, "Low-Temperature Synthesis of Hexagonal (Wurtzite) ZnS Nanocrystals," Journal of the American Chemical Society, Vol. 126, No. 22, 2004, pp. 6874-6875. doi:10.1021/ja048650g

[10] Y. J. Zhang, H. R. Xu and Q. B. Wang, "Ultrathin Single Crystal ZnS Nanowires," Chemical Communications, Vol. 46, No. 47, 2010, pp. 8941-8943. doi:10.1039/c0cc02549f

[11] C. B. Murray, C. R. Kagan and M. G. Bawendi, "Synthesis and Characterization of Monodisperse Nanocrystals and Close-Packed Nanocrystal Assemblies," Annual Review of Materials Science, Vol. 30, No. 1, 2000, pp. 545-610. doi:10.1146/annurev.matsci.30.1.545

[12] R. F. Service, "Don't Sweat the Small Stuff," Science, Vol. 320, 2008, pp. 1584-1585. doi:10.1126/science.320.5883.1584b

[13] P. F. Hu and Y. L. Cao, "A New Chemical Route to a Hybrid Nanostructure: Room-Temperature Solid-State Reaction Synthesis of $\mathrm{Ag} @ \mathrm{AgCl}$ with Efficient Photocatalysis," Dalton Transactions, Vol. 41, No. 29, 2012, pp. 8908-8912. doi:10.1039/c2dt30779k

[14] S. Kulmala and J. Suomi, "Current Status of Modern Analytical Luminescence Methods," Analytica Chimica Acta, Vol. 500, No. 1-2, 2003, pp. 21-69. doi:10.1016/j.aca.2003.09.004

[15] W. G. Becker and A. J. Bard, "Photoluminescence and Photoinduced Oxygen Adsorption of Colloidal Zinc Sulfide Dispersions," The Journal of Physical Chemistry, Vol. 87, No. 24, 1983, pp. 4888-4893. doi: $10.1021 / \mathrm{j} 150642 \mathrm{a} 026$

[16] N. Chestnoy, T. D. Harris, R. Hull and L. E. Brus, "Luminescence and Photophysics of CdS Semiconductor Clusters: The Nature of the Emitting Electronic State," The Journal of Physical Chemistry, Vol. 90, No. 15, 1986, pp. 3393-3399. doi:10.1021/j100406a018 
[17] S. Wageh, Z. S. Ling and X. X. Rong, "Growth and Optical Properties of Colloidal ZnS Nanoparticles," Journal of Crystal Growth, Vol. 255, No. 3-4, 2003, pp. 332-337. doi:10.1016/S0022-0248(03)01258-2

[18] J. H. Yu, J. Joo, H. M. Park, S. Baik, Y. W. Kim, S. C.
Kim and T. Hyeon, "Synthesis of Quantum-Sized Cubic ZnS Nanorods by the Oriented Attachment Mechanism," Journal of the American Chemical Society, Vol. 127, No. 15, 2005, pp. 5662-5670. doi:10.1021/ja044593f 


\section{Characterization}

The crystalline structures of the products were analyzed by a powder X-ray diffractometer (XRD, MXP18AHF, $\mathrm{MAC})$ with $\mathrm{Cu}-\mathrm{K} \alpha$ radiation $(\lambda=0.154056 \mathrm{~nm})$. The morphologies, microstructures, and crystal lattice of the obtained samples were characterized by transmission electron microscopy (TEM, JEM-2010F at $200 \mathrm{KV}$ ). The UV spectra were recorded on a spectrophotometer (HITACHI U-3310) at room temperature. The photoluminescence spectra were obtained by using a HITACHI F-4500 fluorescence spectrophotometer at room temperature.

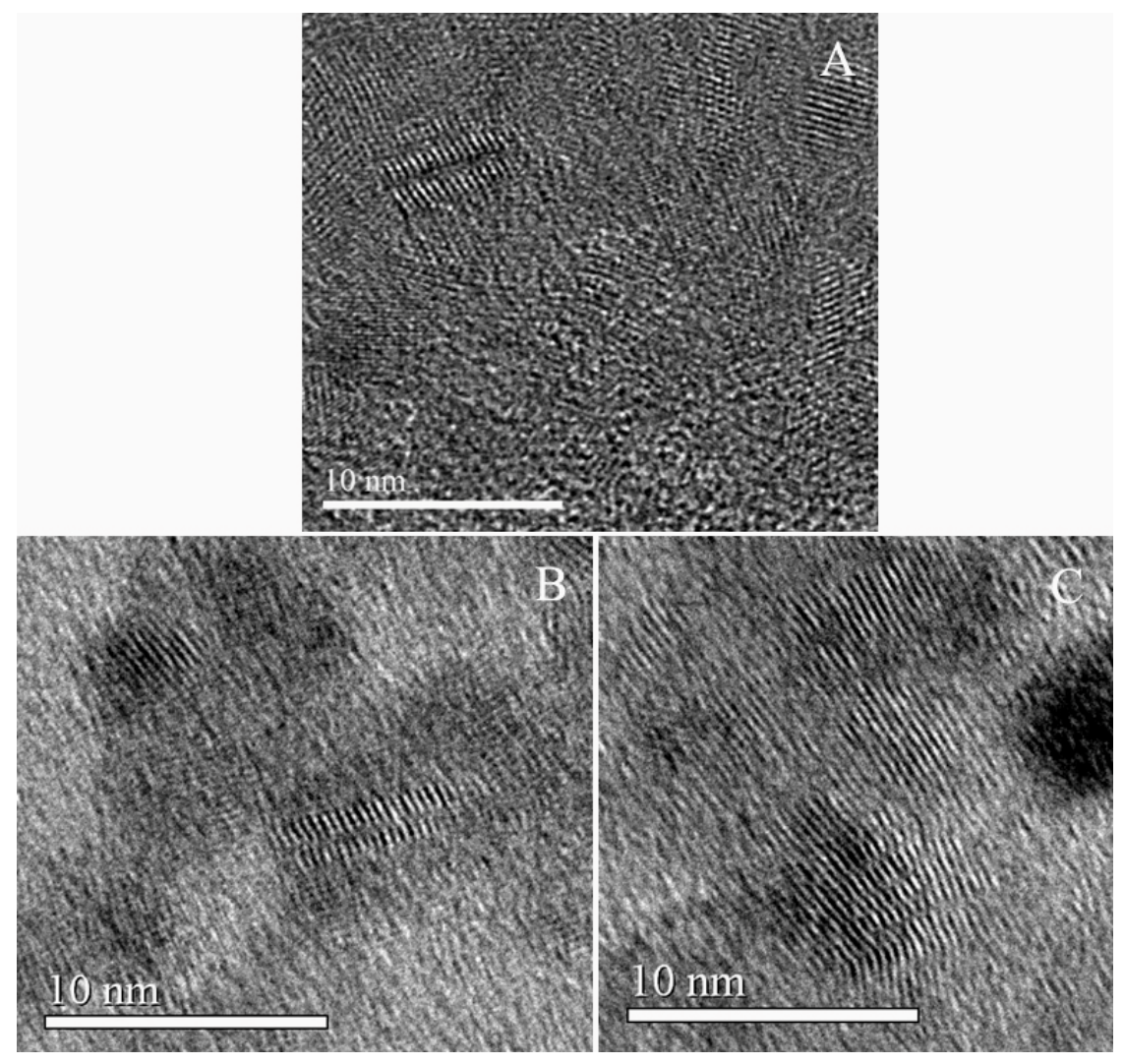

Figure S1. HRTEM images of Q-ZnS-ii nanoparticles with one-dimensional and wave-like structures.

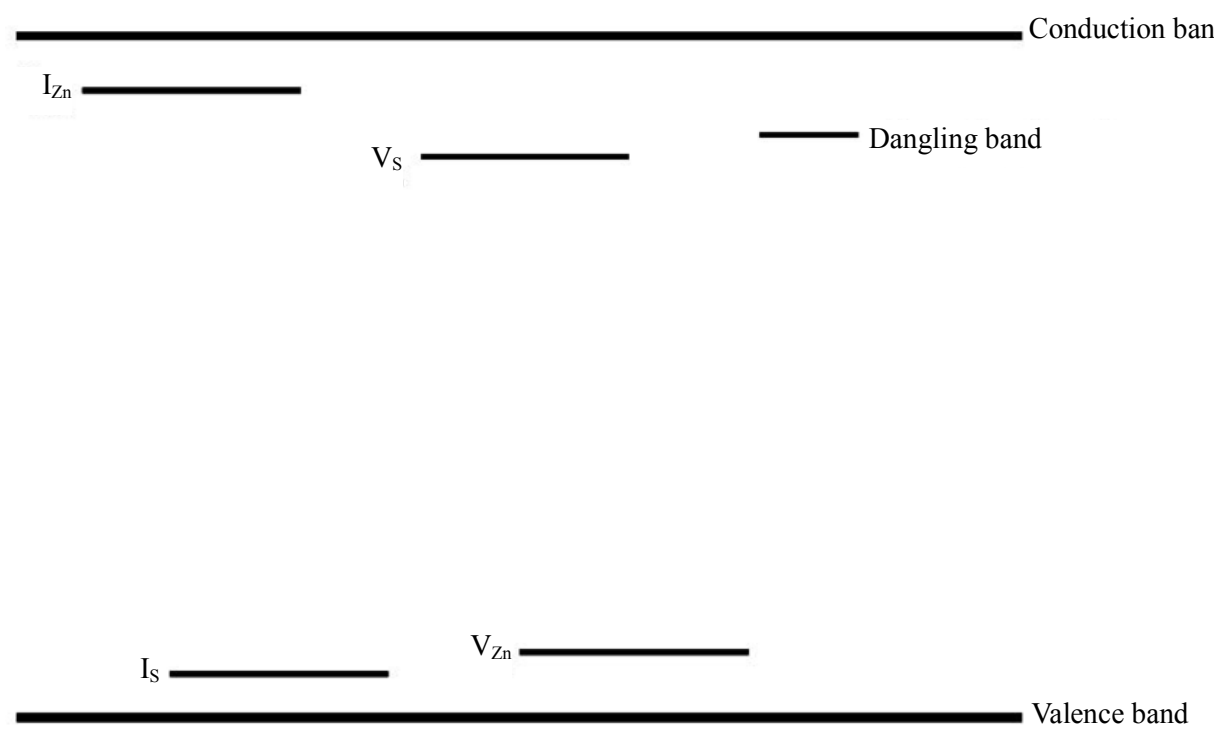

Figure S2. Schematic energy level diagram. 


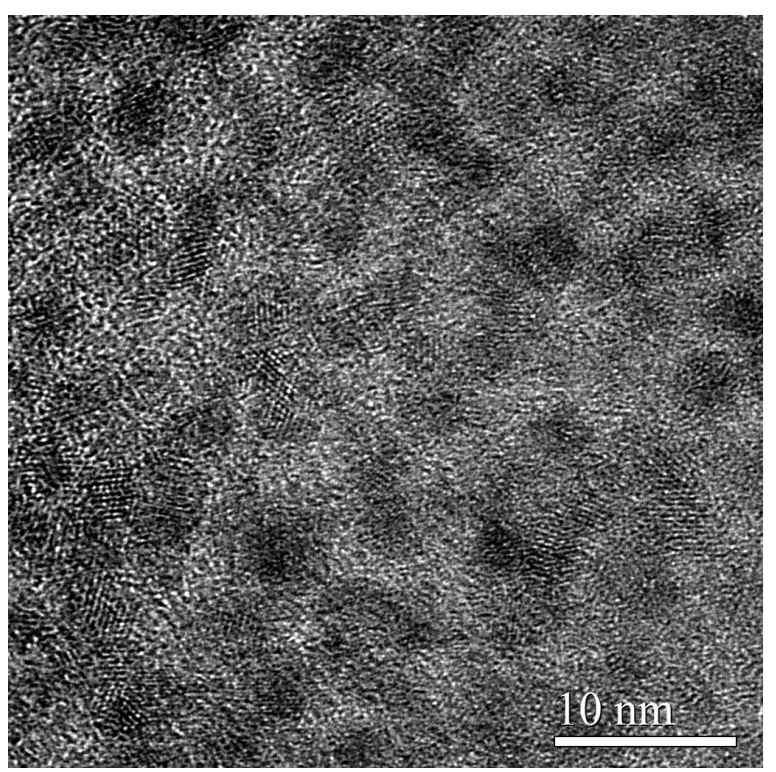

Figure S3. HRTEM image of CdS QDs synthesized through room-temperature solid-state route with SDBS (molar ratio of $1: 10$ for SDBS to zinc salt). 